\title{
A narrative review of the research progress and clinical application of platelet-rich plasma
}

\author{
Yiqiu Cao ${ }^{1,2,3 \#} \wedge$, Xiaoxuan Zhu ${ }^{1,2,3 \#}$, Ruixin Zhou ${ }^{1,2,3 \#}$, Yayi $\mathrm{He}^{1,2}$, Zhourui $\mathrm{Wu}^{1,3}$, Yu Chen ${ }^{4}$ \\ ${ }^{1}$ Medical School, Tongji University, Shanghai, China; ${ }^{2}$ Department of Medical Oncology, Shanghai Pulmonary Hospital, Tongji University, \\ Shanghai, China; ${ }^{3}$ Division of Spine, Department of Orthopedics, Tongji Hospital Affiliated to Tongji University School of Medicine, Shanghai, \\ China; ${ }^{4}$ Spine Center, Orthopedic Department, Shanghai Changzheng Hospital, Shanghai, China \\ Contributions: (I) Conception and design: Y Chen; (II) Administrative support: Y He, Z Wu; (III) Provision of study materials or patients: None; \\ (IV) Collection and assembly of data: X Zhu, R Zhou; (V) Data analysis and interpretation: Y Cao; (VI) Manuscript writing: All authors; (VII) Final \\ approval of manuscript: All authors. \\ "These authors contributed equally to this work. \\ Correspondence to: Yayi He. Department of Medical Oncology, Shanghai Pulmonary Hospital, Tongji University, No. 507, Zhengmin Road, Shanghai \\ 200433, China. Email: 2250601@qq.com; Zhourui Wu. Division of Spine, Department of Orthopedics, Tongji Hospital affiliated to Tongji \\ University School of Medicine, Shanghai 200065, China. Email: wuzhourui@tongji.edu.cn; Yu Chen. Spine Center, Orthopedic Department, \\ Shanghai Changzheng Hospital, No. 415, Fengyang Road, Shanghai 200433, China. Email: yugangg2003@163.com.
}

\begin{abstract}
As a traditional treatment invented in the 1970s, the usage of platelet-rich plasma (PRP) has been reported constantly in many medical areas, such as tissue regeneration, wound healing, ligament repair, hair loss, and so on. In this review, we focus on the administration of PRP in musculoskeletal recovery. As a part of autogenous blood plasma, PRP's platelet concentration is above the baseline. It is extracted from the host's blood sample collected before centrifugal separation. The history, mechanism and preparation of PRP, and existing clinical applications of it will become a helper for clinicians to better understand this therapy. However, the molecular mechanism of PRP treatment is still under debate. On the other hand, because of the safety concern during the PRP's preparation, the practical application of PRP is only applied in many rare cases, especially in spinal diseases. In this paper, we attempt to make a better understanding of the mechanism of PRP and the preparation of PRP; meanwhile, to raise existing questions about further application of PRP in the future. We recommend that PRP should be used in spinal diseases and other fields and in the future we ought to find a safe, simple, and standardized PRP preparation protocol.
\end{abstract}

Keywords: Platelet-rich plasma (PRP); molecular mechanism; musculoskeletal; spinal disease

Submitted Nov 08, 2020. Accepted for publication Jan 21, 2021.

doi: 10.21037/apm-20-2223

View this article at: http://dx.doi.org/10.21037/apm-20-2223

\section{Introduction}

Platelet-rich plasma (PRP) is a biological product obtained from autologous peripheral blood after centrifugation. It has little immune rejection reaction to itself and contains a large number of proteins, such as platelet-derived growth factor (PDGF), transforming growth factor- $\beta$ (TGF- $\beta$ ), insulinlike growth factor (IGF), vascular endothelial growth factor (VEGF), and epidermal growth factor (EGF). These growth factors have the advantage of acceleration of bone marrow mesenchymal stem cells (BMMCs) differentiation and osteoblasts and fibroblasts proliferation. Based on the advantage of PRP, it has been brought into focus in the field of degenerative disc diseases (IDD) experimental researches, especially in terms of degenerative disc repairing

$\wedge$ ORCID: 0000-0003-2838-2453. 
and bone fusion. Some of the research findings have been applied to clinical treatments. In this paper, we mainly raised four questions. What is the history of PRP? What is the molecular mechanism of PRP? What is the clinical application of PRP? And what are the limitations of PRP and the possible prospect of PRP?

We present the following article in accordance with the Narrative Review reporting checklist (available at http:// dx.doi.org/10.21037/apm-20-2223).

\section{Methods}

Search Strategy for PRP. Original English studies published between January 1983 and June 2020 were searched. The electronic databases used for the search were PubMed, EMBASE, Web of Science, the Cochrane Library, EBSCO (CINAHL), Spine journal, BMC, NCBI, Oxford Academic, Science Direct. There were no language restrictions. We conducted a broad search using the following keywords or text words: "PRP", "History of PRP", "PRP devices", "Molecular mechanism of PRP", "Clinical application of PRP", and "Limitations of PRP". We also searched for the following medical subject headings: "PRP".

\section{History of PRP}

PRP was first developed by hematologists in the 1970 s as a blood transfusion product for the treatment of thrombocytopenia (1). In the 1980s, PRP began to be used in maxillofacial surgery due to its positive advantages on anti-inflammatory and cell proliferation (2). Later on, PRP was primarily applied in the musculoskeletal area of sports injuries. The application of PRP has been reported in the rehabilitation among athletes (3). Furthermore, PRP is also used in cardiac, pediatric, gynecology, urology, orthopedic surgery, ophthalmology, and other fields (4). Since the last decade, PRP has also been widely used in dermatology, especially in tissue reproduction, wound-healing, skin regeneration, and hair loss (5-11). PRP was first identified in 2006 as a feasible new treatment for hair loss (12). In recent years, PRP has also been used to treat orthopedic diseases, such as arthritis, and promoting bone fusion $(13,14)$.

\section{PRP preparation}

There are many ways to make PRP, different centrifugal times, centrifugal force, and centrifugal time, PRP produced are different. In addition, the platelet recovery and activation rate in PRP can also be affected by factors such as different length of axis, different diameter and length of the centrifuge tube, the material of centrifuge tube (glass or plastic), and even different body positions or blood drawing at different times of the day. At present, there is commercial equipment to make PRP on the market. The concentrations of platelets, white blood cells, and growth factors are all different in the PRP produced. Since the concentration of growth factor and white blood cells can significantly affect the effect of PRP, the concentration of PRP components produced by the machine is relatively stable, compared with manual production. The key tricky of PRP preparation is to use twice centrifugation method. After the first slow and short centrifugation, the red blood cells (RBCs) with the largest sedimentation coefficient are discarded. Then, with the second high-speed and long centrifugation platelet is collected as many as possible. To obtain a better efficiency of platelet collection, it is necessary to develop a matching centrifuge method, rather than the centrifugal force and centrifuge time reported in the literature (15).

At the same time, there is a study focused on centrifugation times. This study indicated that PRP prepared by the Landesberg method has high platelet concentration and low activation rate (16). Thus, we recommend that the preparation of PRP should follow the centrifugation method two times, and centrifuge the RBCs with the largest sedimentation coefficient to the bottom of the tube for the first time, and then remove the RBCs; the second-high speed long centrifugation is designed to collect as many platelets from the serum as possible to the bottom of the tube.

Simultaneously, there are several studies on platelet concentrations. Three different PRP separation methods were used: a single-spin process yielding a lower platelet concentration (PRPLP), a single-spin process yielding high platelet and white blood cell concentrations (PRPHP), and a double-spin that produces a higher platelet concentration and lower white blood cell concentration (PRPDS). As the result, the "more is better" theory using higher platelet concentrations does not work (17). And in another study, indicated that after PRP extraction, the platelet count should be more than 4 times higher than that of whole blood. Studies have shown that 4-5 times of platelet concentration can effectively promote bone and soft tissue repair, higher concentration does not show better repair effect, and more than 4 times of concentration can be obtained manually, without the need for special preparation instruments (18).

At present, there is no unified preparation standard for 
PRP, and the platelet collection rate of different preparation systems is different due to the great differences in various PRP collection and preparation processes. Usually, will collect whole blood with anticoagulant factors (such as sodium citrate, dextran and ethylenediamine tetraacetic acid) hybrid, and centrifugal separation of RBC, poor plasma (PPP), and contains concentrated platelet "blood sedimentation layer" buffy, remove the RBC and the PPP layer, further separation of platelet concentrate layer. PRP can be injected directly into the affected area or "activated" by adding calcium chloride or thrombin, which degranulates the activated platelets and releases growth factors and differentiation factors. After activation, about $70 \%$ of stored growth factors were released within $10 \mathrm{~min}$, and almost $100 \%$ were released within $1 \mathrm{~h}$. The remaining few growth factors were released within $8-10 \mathrm{~d}$ of platelet survival (19).

\section{Molecular mechanism of PRP}

PRP has seven basic proteins: PDGF, TGF- $\beta$, VEGF, EGF, hepatocyte growth factor (HGF), fibroblast growth factor (FGF), and IGF-1 (20). PDGF is a glycoprotein produced from platelet degranulation beside the injury. It can activate the membrane receptor on the target cell and form a high-energy phosphate bond, thus activating the signaling protein to activate the particular activity of the target cell, including mitogenesis, angiogenesis, and macrophage activation. TGF- $\beta$ is produced by platelets and macrophages and serves as an anti-proliferation factor in natural epithelial cells. Fibroblasts, marrow stem cells, and pre-osteoblasts are the target cells for TGF- $\beta$. VEGF is a signaling protein secreted by cells and activates angiogenesis (21). EGF activates the growth, proliferation, and variation of the cells through binding to the receptor EGFR (22-25).

In the local environment, the presence of bioactive molecules in PRP promotes 4 major roles. They are proliferation, migration, cell differentiation, and angiogenesis. Dermal papilla (DP) cells produce growth factors, such as IGF-1, FGF-7, HGF, and VEGF, which are in charge of keeping hair follicles during the growth period of the hair cycle (26). EGF and TGF are related to managing the growth and differentiation of dilated cells, and PGF is possibly associated with the function of the uplift and the interaction of related tissues from the follicle morphogenesis. PRP can increase angiogenesis, block apoptosis, and extend the time of the growth period (25) (Figure 1).

\section{Clinical application}

As a source of growth factors for many treatments, PRP has become an important clinical therapy. One of the greatest advantages of PRP is that it arrives to the injured area directly. PRP also helps regenerate damaged or worn tissue, reducing back pain and increasing spinal function $(27,28)$.

Chronic knee and joint pain are one of the most common ailments in adults. These injuries include osteoarthritis, tendon strain, rotator cuff injury, plantar fasciitis, sports hernia, cartilage injury of the knee joint, sports injury, etc. PRP has been shown to help promote the growth of cells responsible for making new tissue in damaged areas, whether or not they can make new tendons, muscles, cartilage, and connective tissue. PRP also helps tissues heal more effectively, making them stronger, thicker, and healthier. This is particularly useful for cartilage damage, such as osteoarthritis, because it can help treat the root of the problem by stimulating new cartilage growth, which is often difficult. After all, blood vessels are in short supply (29-31).

In a total knee replacement, PRP was applied to the wound surface, synovial membrane, and wound surface, and inflammation and blood loss were significantly reduced (32). In a variety of cosmetic surgery, the clinical observation of facial edema significantly reduced (33).

Infections of sternal wounds after heart surgery are common, and in some cases, can lead to death. PRP injections have been shown to improve the healing process and reduce the risk of infection. Excessive bleeding can sometimes occur during heart surgery. As a result, some patients need blood transfusions during surgery. The application of PRP in these procedures reduces the total amount of homologous blood a patient needs for transfusion. The use of PRP could also reduce the number of patients requiring additional blood transfusions (34).

Back pain is one of the biggest medical problems, and PRP injections may be a viable solution. Disc degeneration occurs when the discs between each vertebra in the spine begin to wear out, harden, and lose their ability to cope with stress. This can lead to other problems, such as a herniated disc or nerve compression, or injury. PRP helps prevent fluid leakage by closing cracks in the tissue and healing them, thereby helping to suppress the process of disc degeneration.

Li et al. conducted animal studies to show that PRP can promote osteoarthritis cartilage repair in rabbits (35). Wang et al. established 30 rabbits model of arthritis and 


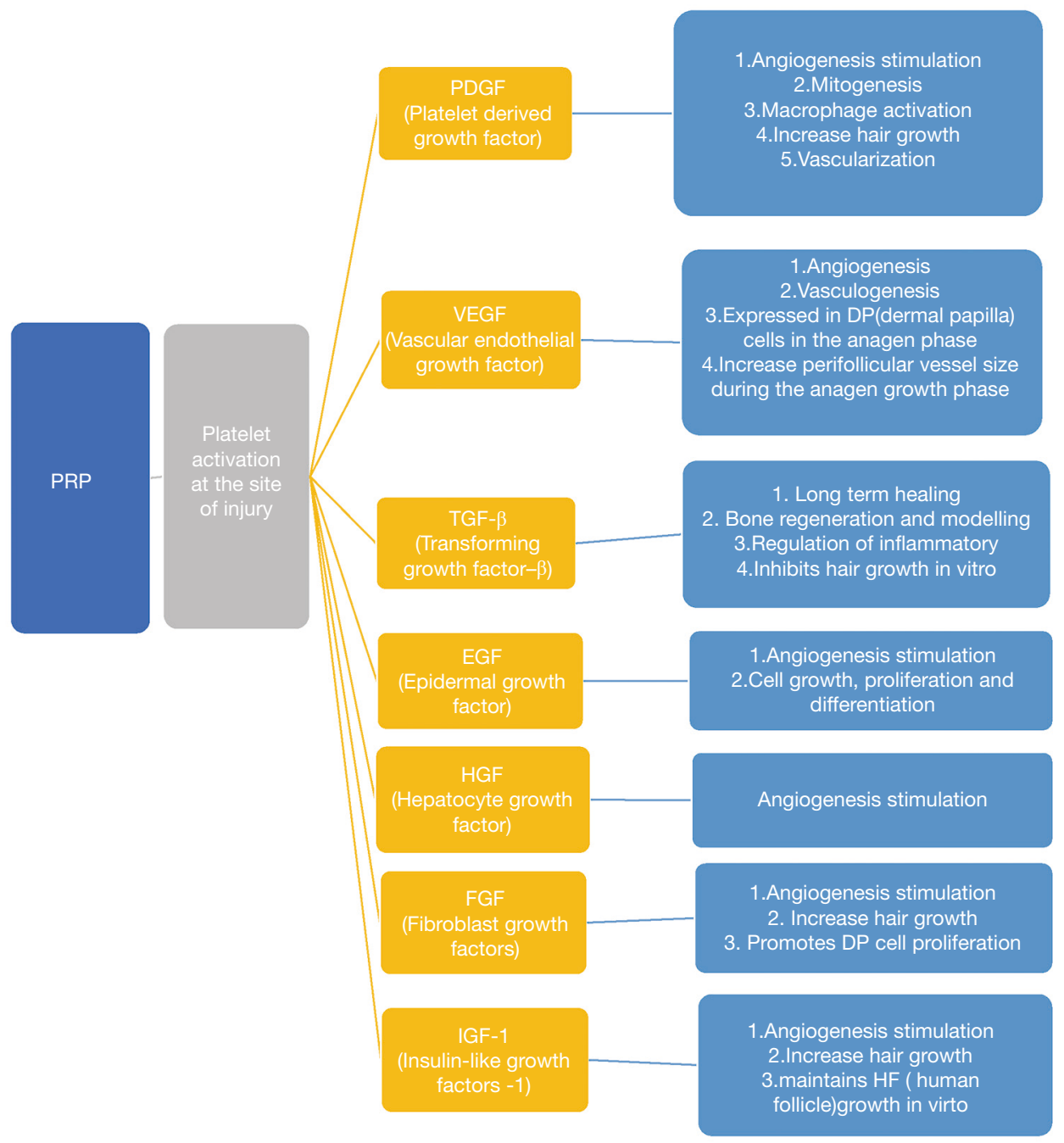

Figure 1 The main functions of platelet-rich plasma (PRP).

verified the effect of PRP on promoting cartilage repair and inhibiting MMP13 (36). Siclari et al. reviewed the 52 cases under the arthroscope knee joint Pridie drilling postoperative patients, 9 months after injection of PRP follow-up found that patients with histological assessment shows homogeneous transparent cartilage repair tissue, including the four years of follow-up, in patients with 21 cartilage repair, 20 patients proved that PRP cartilage repair patients with continuous improvement (37).

The use of enhanced bone and soft tissue regeneration with the application of PRP is suitable for enhancing the relationship between alveolar ridge contours, for desired bridge and mastoid aesthetic fixations, for healthy periodontal attachment of alveolar ridge complexes, and placement of bone implants $(38,39)$. Simultaneously,
PRP is applied to alveolar ridge repair, oral/nasal fistula repair, postoperative hemostasis in the bone graft donor area, continuous defect of the mandible, and surgical hemophilia (40). In sports medicine, PRP is also widely used to recover injured ligaments and tendons without surgery.

\section{Limitations and prospects}

Although PRP has many theoretical functions and certain clinical application value, PRP is not used as a major treatment in practice. Currently, PRP is mainly used for cosmetic or dental treatment. At present, the controversy or disadvantage of PRP mainly lies in the two aspects of infection and abuse (41).

PRP's technology mainly uses platelets for action, which 
requires high requirements for blood separation technology and a sterile environment. The preparation of PRP must be carried out safely by certified perfusionists or other health care professionals who should be trained in suitable aseptic filtration and transfusion techniques that meet safety requirements. In many institutions without conditions and qualifications, beauty salons have bought test tubes, centrifuges have carried out PRP therapy. There's a lot of insecurity involved, like is the environment sterile? Are there any other substances added to it? Is the operator a medical worker? Many of the devices and operations are experimental and cannot be used medically. Can active ingredients be extracted? Can the informal sector tell if you're a good fit for PRP therapy? (42).

PRP extraction and injection processes sometimes require second centrifugation. It can be seen from this process that the degree of manual operation is very high. If the PRP is extracted carelessly in this process, it will be contaminated. If injected into the human body, it may cause infection. If it is a normal operation, to ensure that it is not contaminated, in theory, is safe.

At the same time, whether the problem of centrifugation or extraction will affect the effect of PRP therapy. Is the PRP concentration from the centrifuge high enough, what is its quality, and are platelets destroyed? What we use is a thin layer of blood, and if you take it manually with a needle, is it the plasma, or is it the PRP underneath? It's hard to control. So, some people think that the effect is average, although there is no negative effect, really just average. Only when the concentration is high and the amount is sufficient, can the theoretical effect be expected. Standard sterile operating room and laboratory are the safest premise for PRP extraction (43-45).

Although PRP has been in use for a long time, there is still confusion over how to define, classify, and describe the different variants of platelet concentrate. In addition to the lack of accurate characterization of the products under test in most articles on the subject, there are wide variations in reporting protocols for the standardization and preparation of PRP. In addition, the high cost of PRP kits, which are commercially available, prevents their use in a larger population (46). In the future, our goal should be to find a safe, simple, and standardized PRP preparation protocol, and to follow this protocol to obtain the best stable platelet yield.

\section{Conclusions}

PRP is a biological product obtained from autologous peripheral blood after centrifugation. PRP can be prepared using standard blood bank technology or instant care devices, including blood cell preservation/separator or desktop devices. PRP has seven basic proteins: PDGF, TGF- $\beta$, VEGF, EGF, HGF, FGF, and IGF- 1 . PRP, as a source of growth factors for many treatments, has become an important clinical therapy. Although PRP has many theoretical functions and certain clinical application value, PRP is not used as a major treatment in practice. At present, the controversy or disadvantage of PRP mainly lies in the two aspects of infection and abuse. There are some disputes on the safety of PRP's use, so the practical application of PRP is not common, especially in spinal diseases. At present, there is no unified preparation standard for PRP, in the future, our goal should be to find a safe, simple, and standardized PRP preparation protocol, and to follow this protocol to obtain the best stable platelet yield. We hope that this review serves as a basis for further research on the use of PRP and more and more researchers can pay more attention to the safety concerns of PRP and find a safer preparation.

\section{Acknowledgments}

The authors are thankful for Dr. Camelia Danilov at University of Southern California for critical proofreading the manuscript and providing a lot of valuable advice.

Funding: This study was supported in part by National Natural Science Foundation of China (81802255), Young Talents in Shanghai (2019 QNBJ), 'Dream Tutor' Outstanding Young Talents Program (fkyq1901), Clinical Research Project of Shanghai Pulmonary Hospital (fk18005), Key Discipline in 2019 (oncology), Project of Shanghai Municipal Science and Technology Commission (Project of Municipal Science and Technology Commission), Scientific research project of Shanghai Pulmonary Hospital (fkcx1903), Shanghai Municipal Commission of Health and Family Planning (2017YQ050), Innovation Training Project of SITP of Tongji University, and Key Projects of Leading Talent (19411950300), Shanghai Pujiang Program (No. 19PJ1409200) and the National Natural Science Foundation of China (No. 82071370) to ZW.

\section{Footnote}

Reporting Checklist: The authors have completed the Narrative Review reporting checklist. Available at http:// dx.doi.org/10.21037/apm-20-2223 
Peer Review File: Available at http://dx.doi.org/10.21037/ apm-20-2223

Conflicts of Interest: All authors have completed the ICMJE uniform disclosure form (available at http://dx.doi. org/10.21037/apm-20-2223). The authors have no conflicts of interest to declare.

Ethical Statement: The authors are accountable for all aspects of the work in ensuring that questions related to the accuracy or integrity of any part of the work are appropriately investigated and resolved.

Open Access Statement: This is an Open Access article distributed in accordance with the Creative Commons Attribution-NonCommercial-NoDerivs 4.0 International License (CC BY-NC-ND 4.0), which permits the noncommercial replication and distribution of the article with the strict proviso that no changes or edits are made and the original work is properly cited (including links to both the formal publication through the relevant DOI and the license). See: https://creativecommons.org/licenses/by-nc-nd/4.0/.

\section{References}

1. Andia I, Abate M. Platelet-rich plasma: underlying biology and clinical correlates. Regen Med 2013;8:645-58.

2. Conde Montero E, Fernández Santos ME, Suárez Fernández R. Platelet-rich plasma: applications in dermatology. Actas Dermosifiliogr 2015;106:104-11.

3. Lynch MD, Bashir S. Applications of platelet-rich plasma in dermatology: A critical appraisal of the literature. J Dermatolog Treat 2016;27:285-9.

4. Li ZJ, Choi HI, Choi DK, et al. Autologous platelet-rich plasma: a potential therapeutic tool for promoting hair growth. Dermatol Surg 2012;38:1040-6.

5. Sommeling CE, Heyneman A, Hoeksema H, et al. The use of platelet-rich plasma in plastic surgery: a systematic review. J Plast Reconstr Aesthet Surg 2013;66:301-11.

6. Salazar-Álvarez AE, Riera-del-Moral LF, García-Arranz $\mathrm{M}$, et al. Use of platelet-rich plasma in the healing of chronic ulcers of the lower extremity. Actas Dermosifiliogr 2014;105:597-604.

7. Picard F, Hersant B, Bosc R, et al. Should we use plateletrich plasma as an adjunct therapy to treat "acute wounds," "burns," and "laser therapies": A review and a proposal of a quality criteria checklist for further studies. Wound Repair
Regen 2015;23:163-70.

8. Cobos R, Aizpuru F, Parraza N, et al. Effectiveness and efficiency of platelet rich plasma in the treatment of diabetic ulcers. Curr Pharm Biotechnol 2015;16:630-4.

9. Sclafani AP, Azzi J. Platelet Preparations for Use in Facial Rejuvenation and Wound Healing: A Critical Review of Current Literature. Aesthetic Plast Surg 2015;39:495-505.

10. Kim DH, Je YJ, Kim CD, et al. Can Platelet-rich Plasma Be Used for Skin Rejuvenation? Evaluation of Effects of Platelet-rich Plasma on Human Dermal Fibroblast. Ann Dermatol 2011;23:424-31.

11. Sclafani AP, McCormick SA. Induction of dermal collagenesis, angiogenesis, and adipogenesis in human skin by injection of platelet-rich fibrin matrix. Arch Facial Plast Surg 2012;14:132-6.

12. Giordano S, Romeo M, Lankinen P. Platelet-rich plasma for androgenetic alopecia: Does it work? Evidence from meta analysis. J Cosmet Dermatol 2017;16:374-81.

13. Tarantino R, Donnarumma P, Mancarella C, et al. Posterolateral arthrodesis in lumbar spine surgery using autologous platelet-rich plasma and cancellous bone substitute: an osteoinductive and osteoconductive effect. Global Spine J 2014;4:137-42.

14. Alves R, Grimalt R. A Review of Platelet-Rich Plasma: History, Biology, Mechanism of Action, and Classification. Skin Appendage Disord 2018;4:18-24.

15. Zhang CQ, Yuan T. Advances and controversies in clinical application of platelet-rich plasma. Chinese Journal of Joint Surgery (Electronic Version) 2016;10:588-91.

16. Yuan T, Zhang CQ. Fabrication and principle of plateletrich plasma in the the repair of bone and soft tissues. Chinese Journal of Clinical Rehabilitation 2004;8:7939-41.

17. Mazzocca AD, McCarthy MB, Chowaniec DM, et al. The positive effects of different platelet-rich plasma methods on human muscle, bone, and tendon cells. Am J Sports Med 2012;40:1742-9.

18. Wang Lin, Yang JY, Zhang BW, et al. Platelet-rich plasma injection for the treatment of atrophic fracture nonunion. China Journal of Orthopaedics and Traumatology 2020;33:261-4.

19. Dong WX, Ma Y, Guo Y, et al. Progress in the application of platelet-rich plasma in orthopedic diseases. Zhejiang Medical Journal 2020;42:516-20.

20. Marx RE. Platelet-rich plasma: evidence to support its use. J Oral Maxillofac Surg 2004;62:489-96.

21. Senger DR, Galli SJ, Dvorak AM, et al. Tumor cells secrete a vascular permeability factor that promotes accumulation of ascites fluid. Science 1983;219:983-5. 
22. Herbst RS. Review of epidermal growth factor receptor biology. Int J Radiat Oncol Biol Phys 2004;59:21-6.

23. Gupta AK, Carviel J. A Mechanistic Model of PlateletRich Plasma Treatment for Androgenetic Alopecia. Dermatol Surg 2016;42:1335-9.

24. Uebel CO, da Silva JB, Cantarelli D, et al. The role of platelet plasma growth factors in male pattern baldness surgery. Plast Reconstr Surg 2006;118:1458-66; discussion 67.

25. Takikawa M, Nakamura S, Nakamura S, et al. Enhanced effect of platelet-rich plasma containing a new carrier on hair growth. Dermatol Surg 2011;37:1721-9.

26. Jain R, De-Eknamkul W. Potential targets in the discovery of new hair growth promoters for androgenic alopecia. Expert Opin Ther Targets 2014;18:787-806.

27. Buckwalter JA, Mankin HJ. Articular cartilage: degeneration and osteoarthritis, repair, regeneration, and transplantation. Instr Course Lect 1998;47:487-504.

28. Buckwalter JA, Mankin HJ. Articular cartilage: tissue design and chondrocyte-matrix interactions. Instr Course Lect 1998;47:477-86.

29. Frazer A, Bunning RA, Thavarajah M, et al. Studies on type II collagen and aggrecan production in human articular chondrocytes in vitro and effects of transforming growth factor-beta and interleukin-1beta. Osteoarthritis Cartilage 1994;2:235-45.

30. Pujol JP, Chadjichristos C, Legendre F, et al. Interleukin-1 and transforming growth factor-beta 1 as crucial factors in osteoarthritic cartilage metabolism. Connect Tissue Res 2008;49:293-7.

31. Schmidt MB, Chen EH, Lynch SE. A review of the effects of insulin-like growth factor and platelet derived growth factor on in vivo cartilage healing and repair. Osteoarthritis Cartilage 2006;14:403-12.

32. Marx RE. Platelet-rich plasma (PRP): what is PRP and what is not PRP? Implant Dent 2001;10:225-8.

33. Eppley BL, Pietrzak WS, Blanton M. Platelet-rich plasma: a review of biology and applications in plastic surgery. Plast Reconstr Surg 2006;118:147e-59e.

34. Serraino GF, Dominijanni A, Jiritano F, et al. Platelet-rich plasma inside the sternotomy wound reduces the incidence of sternal wound infections. Int Wound J 2015;12:260-4.

35. Li YH, Qu F, Jin Z. The effects of additional PRP on the rehabilitation prognosis, Harris, NRS, Fernandez Esteve eschar scores in treatment of femoral neck fracture by locking plate. Hebei Medical Journal 2020;42:3276-8.

36. Wang ZZ, Yu F, Yang B, et al. Inhibition effect of plateletrich plasma on matrix metalloproteinase-13 expression in rabbit osteoarthritis cartilage. Journal of Capital Medical University 2020;41:923-8.

37. Siclari A, Mascaro G, Kaps C, et al. A 5-year followup after cartilage repair in the knee using a platelet-rich plasma-immersed polymer-based implant. Open Orthop J 2014;8:346-54.

38. Silva A, Sampaio R. Anatomic ACL reconstruction: does the platelet-rich plasma accelerate tendon healing? Knee Surg Sports Traumatol Arthrosc 2009;17:676-82.

39. Cole BJ, Seroyer ST, Filardo G, et al. Platelet-rich plasma: where are we now and where are we going? Sports Health 2010;2:203-10.

40. Jain NK, Gulati M. Platelet-rich plasma: a healing virtuoso. Blood Res 2016;51:3-5.

41. Kevy SV, Jacobson MS. Comparison of methods for point of care preparation of autologous platelet gel. J Extra Corpor Technol 2004;36:28-35.

42. De Somer F, De Brauwer V, Vandekerckhove M, et al. Can autologous thrombin with a rest fraction of ethanol be used safely for activation of concentrated autologous platelets applied on nerves? Eur Spine J 2006;15:501-5.

43. Landesberg R, Moses M, Karpatkin M. Risks of using platelet rich plasma gel. J Oral Maxillofac Surg 1998;56:1116-7.

44. Martinez-Gonzalez JM, Cano-Sanchez J, GonzaloLafuente JC, et al. Do ambulatory-use Platelet-Rich Plasma (PRP) concentrates present risks? Med Oral 2002;7:375-90.

45. Everts PA, Knape JT, Weibrich G, et al. Platelet-rich plasma and platelet gel: a review. J Extra Corpor Technol 2006;38:174-87.

46. Dhurat R, Sukesh M. Principles and Methods of Preparation of Platelet-Rich Plasma: A Review and Author's Perspective. J Cutan Aesthet Surg 2014;7:189-97.
Cite this article as: Cao Y, Zhu X, Zhou R, He Y, Wu Z, Chen Y. A narrative review of the research progress and clinical application of platelet-rich plasma. Ann Palliat Med 2021;10(4):4823-4829. doi: 10.21037/apm-20-2223 